\title{
THE QUARTIC CURVE AS RELATED TO CONICS*
}

A. B. COBLE

A characteristic feature of the plane curve of even order, $2 n$, is the one-to-one correspondence set up by it between curves of class $n$ and curves of order $n$. Given a fundamental curve of order $2 n$, every curve of class $n$ has a definite polar curve of order $n$, every curve of order $n$ has "associated" with it a definite curve of class $n$ whose polar it is.

In the following this correspondence will be studied with special reference to the quartic. Some simultaneous irrational invariantive forms of the system of two quartics will be considered, the method used being an extension to the ternary domain of that employed by HILBERT $\dagger$ for binary forms. A generalization of the configuration known as the self-polar triangle for $n=1$ will be treated.

The first section will be devoted to an investigation of an error contained in a statement of Clifford, $\ddagger$ an error originally pointed out by Professor MoRLey. The facts there obtained suggested the more general inquiry which follows.

Forms of order $n$ will be denoted in general by $f^{n}, g^{n}$, etc., or symbolically by $\alpha_{x}^{n}, \beta_{x}^{n}, \cdots\left(\alpha_{x} \equiv \alpha_{1} x_{1}+\alpha_{2} x_{2}+\alpha_{3} x_{3}\right)$, those of class $n$, by $F^{n}, G^{n}, \cdots$ or by $a_{\xi}^{n}, b_{\xi}^{n}, \ldots ;$ and $c_{\iota \kappa l}$ will be the general expression for an invariantive form of $f^{4}$ of degree $\iota$, order $\kappa$ and class $l$.

\section{§1. A Special Class of Quartic Curves.}

In the collected works of Clifford, p. 117, the following statement is found : The quartic $f^{4}$ has a contravariant of degree 5 and class $4\left(\phi \equiv c_{504}\right)$, the evectant of the invariant $B \S$ which is possessed of this property, that every conic, $c_{\xi}^{2}$, has a polar as to $f^{4}$ whose polar as to $\phi$ is again $c_{\xi}^{2}$.

We shall prove first

* Presented to the Society in conjunction with results obtained by Professor F. MORLEY, December 27, 1901. Received for publication in present form September 25, 1902.

† Mathematische Annalen, vol. 28 (1887).

$\ddagger$ Collected Works, p. 117 .

\& The invariant $B$ is the determinant of sixth order

$$
\left|a_{\iota \kappa, \iota m}\right| \quad(\iota \kappa, l m=11,22,33,23,31,12)
$$

whose vanishing is the condition that a conic apolar to $f^{4}$ exists. 
(I) For the general quartic, $f^{4} \equiv \alpha_{x}^{4}$ there exists no quartic, $F^{4} \equiv a_{\xi}^{4}$ which has with regard to $f^{4}$ the above described property.

For if $F^{4}$ and $f^{4}$ are so related and $c_{\xi}^{2}$ is an arbitrary line conic, the equation

$$
c_{a}^{2} \alpha_{a}^{2} a_{\xi}^{2} \equiv c_{\xi}^{2}
$$

must hold identically for all values of the $\xi$ 's and $c$ 's. In strictness a factor $\lambda$ should be introduced before $c_{\xi}^{2}$ in (1), a factor, however, necessarily independent of $c_{\xi}^{2}$ and therefore supposed incorporated with the coefficients of $F$.

Equating coefficients of $\xi_{\iota} \xi_{\kappa}$ in (1) we have six equations of the type

$$
c_{a}^{2} \alpha_{a}^{2} a_{\iota \kappa}=c_{\iota \kappa},
$$

which are to be identities in the quantities $c_{\iota \kappa}$. If in each equation of the type (2) we equate coefficients of $c_{m n}$ a system of six non-homogeneous equations is obtained. The determinant of this system in the six unknowns

$$
a_{m n 11}, a_{m n 22}, a_{m n 33}, 2 a_{m n 23}, 2 a_{m n 31}, 2 a_{m m 12}
$$

is the invariant $B$ which for the general quartic is not zero. If the minor of $\alpha_{\iota \kappa, l m}$ in $B$ be denoted by $A_{\iota \kappa, l m}$ the solution of the system gives

where

$$
a_{\iota \kappa, m n}=\frac{A_{\iota \kappa, m n}}{\mu_{\iota \kappa, m n} B}
$$

$$
\begin{array}{lllll}
\mu_{\iota \kappa, m n}=1 & \text { if } & \iota=\kappa & \text { and } & m=n, \\
\mu_{\iota \kappa, m n}=2 & \text { if } & \iota \neq \kappa & \text { or } & m \neq n, \\
\mu_{\iota \kappa, m n}=4 & \text { if } & \iota \neq \kappa & \text { and } & m \neq n .
\end{array}
$$

But the symbolic coefficients $a$ are such that $a_{\iota \kappa, m n}=a_{m n, \iota \kappa}=a_{m \iota, n \kappa}$. Hence must

$$
\frac{A_{\iota \kappa, m n}}{\mu_{\iota \kappa, m n}}=\frac{A_{m n, \iota \kappa}}{\mu_{m n, \iota \kappa}}=\frac{A_{m \iota, n \kappa}}{\mu_{m \iota, n \kappa}} .
$$

Since $B$ is a symmetrical determinant we have

$$
A_{\iota \kappa, l m}=A_{l m, \iota \kappa} \neq A_{\iota \iota, m \kappa} .
$$

Relations (4) then, contrary to hypothesis, do impose conditions on the quartic $f$, and this proves $(\mathrm{I})$. Conditions (4) in full are

$$
\begin{gathered}
A_{1212}=4 A_{1122}, \quad A_{1313}=4 A_{1133}, \quad A_{2323}=4 A_{2233}, \quad A_{1231}=2 A_{1123} \\
A_{1223}=2 A_{2231}, \quad A_{3123}=2 A_{3312}, \quad B \neq 0 .
\end{gathered}
$$


(II) If the quartic $f$ satisfies conditions (5), a quartic $F$, namely $\phi$, the evectant of the invariant $B$, actually exists, which has with $f$ the property ascribed to it by Cufforo.

Evidently $F$ actually exists, for in (4) its coefficients have been so determined that (1) is an identity for both $\xi_{\iota \kappa}$ and $c_{\iota \kappa}$. The contravariant $\phi$ is

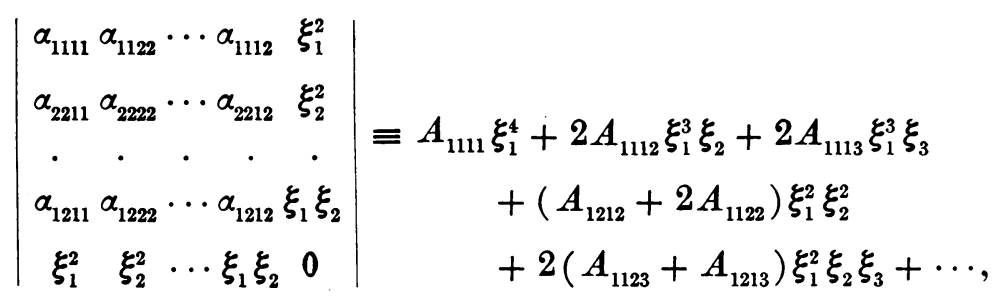

and has been studied by Scherrer. * Applying conditions (5) to the expanded form of $\phi$ the coefficient of $\xi_{\iota} \xi_{k} \xi_{m} \xi_{n}$ becomes the previously determined $a_{\iota \kappa m n}$ of $F$ to within the factor $B$.

An analogous property which does belong to the general quartic is this:

(III) The cubic polar of any point as to the general quartic $f$ has a linear polar as to $\phi$ which is the original point.

For the proof, see $\S 2$, at the end.

A quartic $f$ satisfying conditions (5) will be called a "special" quartic and some of the properties of such quartics will now be derived. Let first

$$
\frac{A_{i j, \kappa l}}{\mu_{\iota j, \kappa l}} \equiv A_{\imath j, \kappa l}^{\prime}
$$

The quartic envelope $\phi$ may then be written symbolically $A_{\xi}^{\prime 4}$.

* Annali, ser. 2, vol. 10. If the polar of $c_{\xi}^{2}$ as to $f^{4}$ is $\gamma_{x}^{2}, c_{\xi}^{2}$ will be called the conic " associate" to $\gamma_{x}^{2}$. If the polar of $c_{\xi}^{2}$ as to $f^{4}$ is the line $\eta$ taken twice, $c_{\xi}^{2}$ will be called the conic " aseociate" to the line $\eta$.

SCHERRER shows that, for a given $\eta$, the form

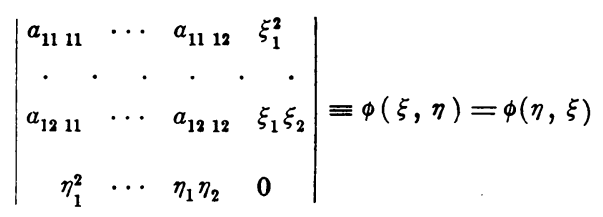

gives the conic associate to the line $\eta$. Also $\phi(\xi, \gamma)$ gives the conic associate to the conic $\gamma_{x}^{2}$.

Therefore $\phi$, or $\phi(\xi, \xi)$, is the locus of lines which touch their own associate conics (selfassociate lines).

It is easily seen that the locus of lines whose associate conics are apolar to a given point conic is the associate of the point conic.

Hence $c_{a}^{2} a_{x}^{2}$ has a polar as to $\phi(\xi, \eta)$ ( $\eta$ variable) which is $B c_{\xi}^{2}$. That is, the form $\phi(\xi, \eta)$ has a property similar to that required of $a_{\xi}^{4}$ by CLIFFORD, as may be seen also from an equation formed analogously to (3). 
(IV) If $f$ is a special quartic, the relation of $f$ and $\phi$ is reciprocal, $i$. e., the $\phi$ of $\phi$ is $f$.

For $\phi$ being $A_{\xi}^{\prime 4}$ we may write the $\phi$ of $\phi$ in determinant form. The $A^{\prime \prime s}$ may then be changed into the $A$ 's by multiplying the last three rows and columns of the determinant by 2 . Since the minor of $A_{\imath j, k l}$ is $B^{4} \alpha_{\imath j, \kappa l}$, we have on developing,

$$
\phi \text { of } \phi=\frac{B^{4}}{64} f .
$$

The reciprocity between $f$ and $\phi$ becomes more marked with the study of their allied forms. Let $\psi$ be the envelope of lines whose associate* conics degenerate into a pair of points which lie on a locus $S$ and let $D$ be the envelope of lines into which the polar conics of Hessian points break up. Then from the definition of $f$ as a special quartic and of the various loci and envelopes we have at once :

(V) If $f$ is a special quartic the Hessian of $\phi$ is the $\psi$ of $f$ and the $\psi$ of $\phi$ is the Hessian of $f$; also the $D$ of $\phi$ is the $S$ of f and the $S$ of $\phi$ is the $D$ of $f$.

Theorems (IV) and (V), exemplifying the reciprocity between $f$ and $\phi$, may be brought under a more general theorem. For this we denote by $I$ any invariantive form associated to a quantic $Q$ given in point (or line) coördinates, line and point coördinates being supposed interchanged in $I$ if $Q$ is given in line (or point) coördinates. We have then

(VI) If $f$ is a special quartic and if the $I_{1}$ of $\phi$ is the $I_{2}$ of $f$ then the $I_{2}$ of $\phi$ is the $I_{1}$ of $f$ to within a factor $\left(B^{4} / 64\right)^{n}$ where $n$ is the degree of $I_{1}$ in the coefficients of $\phi$.

For, taking $\phi$ in the form $A_{\xi}^{\prime 4}$, the $I_{1}$ of $\phi$ is a certain function of the $A^{\prime \prime}$ 's, $x$ 's and $\xi$ 's. The $I_{2}$ of $f$ is what the $I_{1}$ of $\phi$ becomes when the $A^{\prime \prime}$ 's are written in terms of the $\alpha$ 's. Hence the $I_{2}$ of $\phi$ is what the $I_{1}$ of $\phi$ becomes when in each $A_{m n, p q}^{\prime}$ of $I_{1}, \alpha_{\iota j k l}$ is replaced by $A_{\iota j k l}^{\prime}$ (line and point coördinates being interchanged). Thus $A_{m n, p q}^{\prime}$ becomes $B^{4} \alpha_{m n, p q} / 64$.

We shall now prove

(VII) The necessary and sufficient condition that $f$ be a special quartic is the identical vanishing of a certain covariant conic $C$ of degree 5 .

Conditions (5) have already been found to be necessary and sufficient. They may be shown to be identical with the condition of (VII). The general quartic $f$ has two independent $C_{520}, C_{1}$ and $C_{2}$, the first being obtained by operating with $\sigma \dagger\left(C_{204}\right)$ on $H \dagger\left(C_{360}\right)$; the second by operating with $T \nmid\left(C_{306}\right)$ on $f^{2}$. The sought conic $C$ will be a linear combination of $C_{1}$ and $C_{2}$.

For simplicity let $f$ be referred to a triangle of reference chosen as follows : Take for vertex $\xi_{3}$ a point of intersection of the Hessian, $H$, of $f$ and the

* See definition in the preceding footnote.

$\cdot \dagger \sigma \equiv(a \beta \xi)^{4} ; H=$ Hessian $; T \equiv(a \beta \xi)^{2}(\beta \gamma \xi)^{2}(\gamma a \xi)^{2}$. 
Steinerian, $\Sigma$, of $f$. Take for $\xi_{2}$ the point of $\Sigma$ which corresponds to $\xi_{3}$ considered as a point on $H$; and for $\xi_{1}$ the point of $H$ which corresponds to $\xi_{3}$ considered as a point on $\Sigma$. Then

$$
\xi_{2} \xi_{3}^{2}:: f \quad \text { and } \quad \xi_{3} \xi_{1}^{2}:: f
$$

(:: is a symbol for the expression "is apolar to").

The quartic must take the form

$$
\begin{aligned}
f \equiv a x_{1}^{4}+b x_{2}^{4}+c x_{3}^{4}+6 h x_{1}^{2} x_{2}^{2}+12 m x_{1} x_{2}^{2} x_{3} & +4 a_{2} x_{1}^{3} x_{2} \\
& +4 b_{1} x_{2}^{3} x_{1}+4 b_{3} x_{2}^{3} x_{3}+4 c_{1} x_{3}^{3} x_{1} .
\end{aligned}
$$

We find then for the coefficient of $x_{1}^{2}$ in $C_{1}$,

$-a^{2} c b_{3}^{2}+6 a c m a_{2} b_{3}-9 a c h m^{2}-9 a b h c_{1}^{2}+32 a m^{3} c_{1}+8 a b_{1}^{2} c_{1}^{2}$

and for the coefficient of $x_{1}^{2}$ in $C_{2}$,

$$
+5 h^{3} c_{1}^{2}-12 h a_{2} b_{1} c_{1}^{2}+8 b a_{2}^{2} c_{1}^{2},
$$

$-19 a^{2} c b_{3}^{2}+2 a b_{1}^{2} c_{1}^{2}-21 a b h c_{1}^{2}+114 a c m a_{2} b_{3}-21 a c h m^{2}+8 a m^{3} c_{1}$

$$
+72 h a_{2} b_{1} c_{1}^{2}+2 b a_{2}^{2} c_{1}^{2}-55 h^{3} c_{1}^{2}-150 \mathrm{~cm}^{2} a^{2} \text {. }
$$

Let now $C$ be defined* as follows :

$$
150 C=19 C_{1}-C_{2} .
$$

The coefficient of $x_{1}^{2}$ in $C$ is then

$$
b a_{2}^{2} c_{1}^{2}-a c h m^{2}-a b h c_{1}^{2}+4 a m^{3} c_{1}+a b_{1}^{2} c_{1}^{2}+h^{3} c_{1}^{2}-2 h a_{2} b_{1} c_{1}^{2}+c m^{2} a_{2}^{2} .
$$

But this is equal to $A_{2323}-4 A_{2233}$. For the coefficient of $x_{2} x_{3}$ in $C$ a similar calculation gives $2\left(2 A_{1123}-A_{1213}\right)$. We have then

$$
\begin{aligned}
C & \equiv\left(A_{2323}-4 A_{2233}\right) x_{1}^{2}+\left(A_{3131}-4 A_{3311}\right) x_{2}^{2}+\left(A_{1212}-4 A_{1122}\right) x_{3}^{2} \\
& +2\left(2 A_{1123}-A_{1213}\right) x_{2} x_{3}+2\left(2 A_{2231}-A_{1223}\right) x_{3} x_{1}+2\left(2 A_{3312}-A_{2331}\right) x_{1} x_{2} .
\end{aligned}
$$

This form of $C$ renders (VII) evident.

(VIII) If $f$ is a special quartic the two covariant conics of the fifth degree are identical.

This conic $C$ possesses other interesting properties.

(IX) If the quartic $f$ has an apolar conic, $C$ is the apolar conic expressed in point coördinates.

For if $f$ has an apolar conic, $B=0$ and six quantities $p_{\iota \kappa}$ can be determined such that $A_{\imath j, \kappa l}=p_{\imath j} p_{k l}$. Moreover (Clebsch, Crelle's Journal, vol. 49),

\footnotetext{
* The only previous mention of C occurs in Salmon (Higher Plane Curves) where it is merely described and used as a particularly simple combination of the covariants $C_{1}$ and $C_{2}$.
} 


$$
\phi=\left(p_{11} \xi_{1}^{2}+p_{23} \xi_{2} \xi_{3}+\cdots\right)^{2}
$$

is the square of the apolar conic. The equation of the latter in point coördinates is

or

$$
x_{1}^{2}\left(\frac{1}{4} p_{23} p_{23}-p_{22} p_{33}\right)+\cdots+2 x_{2} x_{3}\left(\frac{1}{2} p_{11} p_{23}-\frac{1}{4} p_{12} p_{13}\right)+\cdots,
$$

$$
\text { 1 }\left[\left(A_{2323}-4 A_{2233}\right) x_{1}^{2}+\cdots+2\left(2 A_{1123}-A_{1213}\right) x_{2} x_{3}+\cdots\right] \text {, }
$$

i. e., it is the $C$ found above. We have further

The necessary and sufficient conditions that $f$ have a triple point are $B=0$ and $C \equiv 0 .^{*} \quad \phi$ is then the triple point taken four times.

Let a conic whose polar as to $f$ has a polar as to $\phi$ which is the original conic be called a "fixed" conic ( $f$ no longer being taken as a special quartic). Recalling that the conic "associate" to a given point conic $\gamma_{x}^{2}$ is obtained by replacing the column of $\xi$ 's in the determinant expression for $\phi$ by the coeffcients $\gamma$, a formula may be derived which will indicate some possible variations in the number of fixed conics.

If the combination : $\frac{1}{2}$ (polar of $\gamma$ as to $\left.\phi\right)-6$ (associate of $\gamma$ as to $f$ ) be formed, we obtain

$$
\begin{array}{r}
\xi_{1}^{2}\left[\left(A_{1212}-4 A_{1122}\right) \gamma_{22}+\left(A_{3131}-4 A_{1133}\right) \gamma_{33}+2\left(A_{1231}-2 A_{1123}\right) \gamma_{23}\right]+\cdots \\
+2 \xi_{2} \xi_{3}\left[\left(A_{1213}-2 A_{1123}\right) \gamma_{11}-\left(A_{2323}-4 A_{2233}\right) \gamma_{23}-\left(A_{3123}-2 A_{3312}\right) \gamma_{31}\right. \\
\left.-\left(A_{2312}-2 A_{2231}\right)\right]+\cdots
\end{array}
$$

This resulting conic is the "intermediate" of $\gamma$ and $C$. The intermediate of two conics $\alpha_{x}^{2}$ and $\beta_{x}^{2}$ is $(\alpha \beta \xi)^{2}$, the locus of lines cutting the two conics in harmonic pairs of points. We have then

(XI) For the general quartic $f$ and any conic $\gamma_{x}^{2}$ the following relation holds :

$$
(\gamma \text { on } \phi)=2 I_{\gamma}+12 A_{\gamma} \dagger,
$$

where $I_{\gamma}$ is the intermediate of $\gamma$ and $C, A_{\gamma}$ is the associate of $\gamma$.

If $I_{\gamma} \equiv 0$ or if $I_{\gamma} \equiv \mu A_{\gamma}$ then $(\gamma$ on $\phi) \equiv \lambda A_{\gamma}$, i. e., $\gamma$ is a fixed conic. But in general $I_{\gamma} \neq \mu A_{\gamma}$; hence if every conic $\gamma$ is to be a fixed conic, $I_{\gamma} \equiv 0$ or $C \equiv 0$, a verification of (VII).

The general quartic has, as we shall see later, six fixed conics. Since $C$ 丰 0 , for these conics $I_{\gamma} \equiv \mu A_{\gamma}$. We have then as a first consequence of formula (7),

$1^{\circ}$ The six fixed conics of the general quartic $f$ with regard to $\phi$ are the intermediates of their polars as to $f$ with $C$.

From (7) follows also a proposition whose dual is stated by ScHERRER (loc. cit.):

* Actually only 4 conditions, since the covariant conic furnishes only 3 independent relations. $\dagger$ " $\gamma$ on $\phi$ " is the polar of $\gamma$ with regard to $\phi$. 
$2^{\circ}$ The third polar of a line $L$ as to $\phi$ is the polar point of $L$ to its associate conic.

The following facts also may be derived from (7):

$3^{\circ}$ If $f$ has a cusp the cuspidal tangent is a double line of $\phi$ (possibly an inflexional tangent).

$4^{\circ}$ If $\phi$ has a double line $L, L$ touches also $T$, $\psi$ and $C_{12}{ }^{*}{ }^{*}$

The condition that $C$ degenerate is the vanishing of SALMoN's invariant $E_{1}$, by definition.

$5^{\circ}$ If the invariant $E_{1}$ of the quartic $f$ vanishes there is a pencil of fixed conics.

For if $C$ is the two lines $\alpha \beta$ meeting at a point $P$, any two lines through $P$ and harmonic with $\alpha$ and $\beta$ form a conic whose intermediate as to $\alpha \beta$ vanishes. These conics form a pencil which includes $\alpha^{2}$ and $\beta^{2}$ and may be written $\alpha^{2}+\lambda \beta^{2}$. The associate pencil is $A_{a^{2}}+\lambda A_{\beta^{2}}$.

$6^{\circ}$. If $C$ is the square of a line $L, a$ web of fixed conics exists.

For then the $I_{\gamma}$ for any conic $\gamma$ is the two points in which $\gamma$ meets $L$. If in particular $\gamma \equiv L M$, where $M$ is any line of the plane, $I_{\gamma} \equiv 0$, whence it follows that $L M$ is a fixed conic. The web $A_{L M}$ (projective to the net of associate conics) contains a single infinity of pairs of points $a b$ such that the polar of $a b$ as to $f$ has $L$ for a part. These points $a b$ are then "conjugate points on $G_{L}$ " a covariant curve of third order and degree defined originally by CaPORALI $\dagger$ as the Jacobian of the net of polar conics of couples of points of $L$.

Besides the conics of the web a finite number of other fixed conics exists, viz: the pairs of "conjugate points as to $f$ " which lie on $L$. For by definition $\dagger$ a pair of conjugate points as to $f$ is a pair which lie on their polar conic $\gamma$. If the pair also lie on $L, \gamma$ meets $L$ in this pair, i. e., $I_{\gamma}$ and $A_{\gamma}$ are each this pair of points and $(\gamma$ on $\phi)=\lambda A_{\gamma}$.

\section{§2. Some Simultaneous Irrational Invariantive. Forms of the System $: f^{n}, F^{n}$}

The discussion in the preceding section of the number of fixed conics associated to $f^{4}$ and its contravariant $\phi$ suggests this more general problem :

Given a quartic $f \equiv \alpha_{x}^{4}$ and a quartic $F=a_{\xi}^{4}$, do there exist and if so, in what number, conics whose polars as to $f$ have each a polar as to $F$ identical with the original conic?

Such conics will also be called "fixed" conics. If $c_{\xi}^{2}$ is a fixed conic it must satisfy the identical equation

$$
c_{a}^{2} \alpha_{a}^{2} a_{\xi}^{2} \equiv \lambda c_{\xi}^{2}
$$

Equating coefficients of $\xi$ we have six homogeneous equations in the six unknowns $c_{\iota \kappa}$ whose determinant must then vanish, i. e.,

* Defined by Scherrer, loc. cit.

†Caporali, Memorie di Geometria, pp. 344-5. 


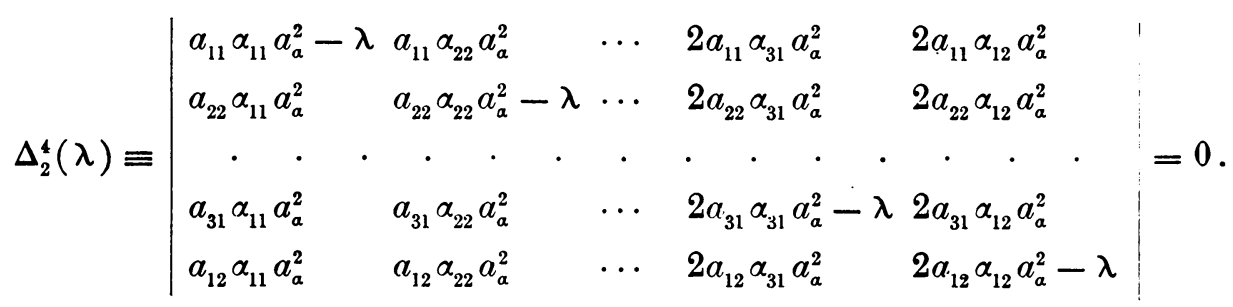

The notation $\Delta_{2}^{4}(\lambda)$ indicates that the $\lambda$-equation is formed with regard to the fixed conics of quartics. Since $f$ and $F$ are given as general, $\Delta_{2}^{4}(\lambda)=0$ must be viewed as a sextic in $\lambda$ with roots $\lambda_{1}, \lambda_{2}, \ldots, \lambda_{6}$ such that any root put for $\lambda$ in (1) makes the resulting system consistent for the determination of the ratios of the coefficients $c_{\iota \kappa}$ which will satisfy (1).

Allowing $f$ and $F$ to interchange their roles, $\Delta_{2}^{4}(\lambda)$ is unaltered ; therefore to a root $\lambda_{\iota}$ corresponds also a point conic which is a fixed conic of $F$ and $f$. Call the six fixed line conics, $c_{\xi: 1}^{2}, \cdots, c_{\xi: 6}^{2}$, the fixed point conics, $\gamma_{x: 1}^{2}, \cdots, \gamma_{x: 6}^{2}$ such that $c_{\xi: \iota}^{2}$ and $\gamma_{x: \iota}^{2}$ each correspond to the root $\lambda_{\iota}$. If the polar of $c_{\xi: \iota}^{2}$, as to $f$ be called $\bar{\gamma}_{x: \iota}^{2}$, then $\bar{\gamma}_{x: \iota}^{2}$, has for polar as to $F, \lambda_{\iota} c_{\xi: \iota}^{2}$, which again has for polar as to $f, \lambda_{\iota} \bar{\gamma}_{x: \iota}^{2}$. Hence $\bar{\gamma}_{x: \iota}^{2} \equiv \gamma_{x: \iota}^{2}$ or $\gamma_{x: \iota}^{2}$ is the polar of $c_{\xi: \iota}^{2}$ as to $f$. Similarly $c_{\xi: \iota}^{2}$ is the polar of $\gamma_{x: \iota}^{2}$ as to $F$.

(I) Corresponding to each root of the equation $\Delta_{2}^{4}(\lambda)=0$, there exist two

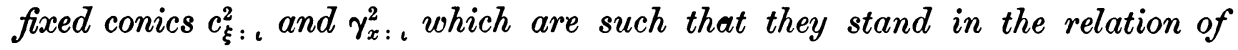
pole and polar to both $f$ and $F$.

The forms $c_{\xi: \text {, and }}^{2} \gamma_{x: \kappa}^{2}$ are defined by the identities

$$
c_{a: \iota}^{2} a_{a}^{2} a_{\xi .}^{2} \equiv \lambda_{\iota} c_{\xi: \iota}^{2}, \quad \gamma_{a: \kappa}^{2} a_{a}^{2} \alpha_{x}^{2} \equiv \lambda_{\kappa} \gamma_{x: \kappa}^{2} .
$$

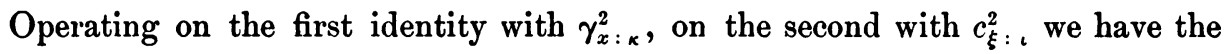
identities

Subtracting,

$$
c_{a: \iota}^{2} a_{a}^{2} \gamma_{a: \kappa}^{2} \equiv \lambda_{\iota} c_{\gamma: \iota \kappa}^{2}, \quad \gamma_{a: \kappa}^{2} a_{a}^{2} c_{a: \iota}^{2} \equiv \lambda_{\kappa} c_{\gamma: \iota \kappa}^{2} \text {. }
$$

$$
0=\left(\lambda_{\iota}-\lambda_{\kappa}\right) c_{\gamma: \iota \kappa}^{2} \text {. }
$$

In general $\lambda_{\iota} \neq \lambda_{\kappa}$ for $\iota \neq \kappa$, therefore $c_{\gamma: \iota \kappa}^{2}=0$.

(II) Among the fixed conics of the general quartics $f$ and $F$ exist the following apolarity relations : $c_{\xi: \iota}^{2}:: \gamma_{x: \kappa}^{2}(\iota \neq \kappa)$.

Suppose now that a linear identity exists:

$$
\rho_{1} c_{\xi: 1}^{2}+\cdots+\rho_{6} c_{\xi: 6}^{2} \equiv 0 .
$$

If we multiply the identity (1) for $\lambda=\lambda_{\iota}$ by $\rho_{\iota}$ and sum for $\iota=1,2, \ldots, 6$ we have by virtue of (2) the identity

$$
\lambda_{1} \rho_{1} c_{\xi: 1}^{2}+\cdots+\lambda_{6} \rho_{6} c_{\xi: 6}^{2} \equiv 0 .
$$


In the same way we obtain four more identities. These can subsist only if the determinant

$$
\left|\begin{array}{cccc}
1 & 1 & \cdots & 1 \\
\lambda_{1} & \lambda_{2} & \cdots & \lambda_{6} \\
\cdot & \cdot & \cdot & \cdot \\
\lambda_{1}^{5} & \lambda_{2}^{5} & \cdots & \lambda_{6}^{5}
\end{array}\right|=\prod_{\imath \neq \kappa}\left(\lambda_{\imath}-\lambda_{\kappa}\right)=0
$$

i. e., only if two roots of $\Delta_{2}^{4}(\lambda)$ are equal.

(III) Among the fixed conics of the general quartics $f$ and $F$ can exist no linear identities.

It follows then that $c_{\xi: \iota}^{2}$ is not :: $\gamma_{x: \iota}^{2}(\iota=1,2, \cdots, 6)$.

(IV) The coefficients of the different powers of $\lambda$ in the equation $\Delta_{2}^{4}(\lambda)=0$ are simultaneous invariants of $f$ and $F$, i. e., the roots $\lambda_{\iota}$ are simultaneous irrational invariants of $f$ and $F$.

Proof of this theorem is obtained from the equation defining $\lambda$, and will be supplied so readily that we may omit it here; so, too, the proof of the following theorem.

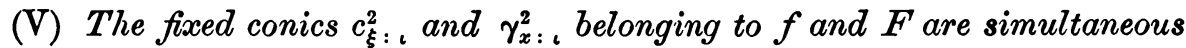
irrational covariants and contravariants of $f$ and $F$.

The equation $\Delta_{2}^{4}(\lambda)=0$ arranged in powers of $\lambda$ may be written

$$
\Delta_{2}^{4}(\lambda) \equiv \lambda^{6}-I_{1} \lambda^{5}+I_{2} \lambda^{4}-I_{3} \lambda^{3}+I_{4} \lambda^{2}-I_{5} \lambda+I_{6}=0,
$$

where $I_{\kappa}$ is a simultaneous invariant of $f$ and $F$ of degree $\kappa$ in the coefficients of each. A general expression for $I_{\kappa}$ may readily be obtained. For this we use temporarily a new notation. Let $g \equiv a_{\xi}^{2} \alpha_{x}^{2}=0$ be a general connex * $(2,2)$ where $\alpha_{\iota \kappa} a_{l m}$ represents the arbitrary coefficient of $x_{\iota} x_{\kappa} \xi_{l} \xi_{m}$. The polar of $c_{\xi}^{2}$ as to $g$ is $c_{a}^{2} a_{\xi}^{2}$. If $c_{a}^{2} \alpha_{\xi}^{2} \equiv \lambda c_{\xi}^{2}$ we have the analogue of the fixed conic previously treated, and $\lambda$ will then satisfy an equation $\Delta(\lambda)=0$ which may be obtained from $\Delta_{2}^{4}(\lambda)$ by suppression of the symbolic factor $a_{a}^{2}$.

Writing $g \equiv a_{\xi}^{2} \alpha_{x}^{2}=b_{\xi}^{2} \beta_{x}^{2}=c_{\xi}^{2} \gamma_{x}^{2}=\cdots$ we have first that a seven-rowed determinant vanishes identically :

$$
D \equiv\left|\begin{array}{cccccc}
a_{a}^{2} & a_{\beta}^{2} & a_{\gamma}^{2} & \cdots & a_{\zeta}^{2} & a_{\xi}^{2} \\
b_{a}^{2} & b_{\beta}^{2} & b_{\gamma}^{2} & \cdots & b_{\zeta}^{b} & b_{\xi}^{2} \\
c_{a}^{2} & c_{\beta}^{2} & c_{\gamma}^{2} & \cdots & c_{\zeta}^{2} & c_{\xi}^{2} \\
\cdot & \cdot & \cdot & \cdot & \cdot & \cdot \\
f_{a}^{2} & f_{\beta}^{2} & f_{\gamma}^{2} & \cdots & f_{\zeta}^{2} & f_{\xi}^{2} \\
\alpha_{x}^{2} & \beta_{x}^{2} & \gamma_{x}^{2} & \cdots & \zeta_{x}^{2} & x_{\xi}^{2}
\end{array}\right| \equiv 0 \text { (identity in } x \text { and } \xi \text { ) }
$$

* The author hopes in future to treat some analogous problems for the general connex by the method of section 4 . 
since there must be a linear identity among any seven ternary quadrics. If now we write for the $\kappa$-rowed determinant

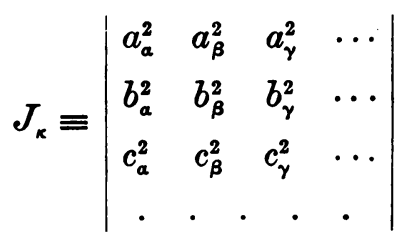

we have, taking into account the permutability of symbols,

$$
D \equiv x_{\xi}^{2} J_{6}-\frac{6 !}{5 !} a_{\xi}^{2} \alpha_{x}^{2} J_{5}+\frac{6 !}{4 !} a_{\xi}^{2} \alpha_{b}^{2} \beta_{x}^{2} J_{4}-\frac{6 !}{3 !} a_{\xi}^{2} \cdots \gamma_{x}^{2} J_{3}+\frac{6 !}{2 !} a_{\xi}^{2} \cdots \delta_{x}^{2} J_{2}
$$

$$
-\frac{6 !}{1} a_{\xi}^{2} \cdots \epsilon_{x}^{2} J_{1}+6 ! a_{\xi}^{2} \cdots \zeta_{x}^{2} \equiv 0
$$

Since $c_{a}^{2} a_{\xi}^{2} \equiv \lambda c_{\xi}^{2}$,

$$
c_{\alpha}^{2} a_{\beta}^{2} b_{\xi}^{2} \equiv \lambda^{2} c_{\xi}^{2}, \quad c_{\alpha}^{2} a_{\beta}^{2} b_{\gamma}^{2} c_{\xi}^{2} \equiv \lambda^{3} c_{\xi}^{2},
$$

Replacing, then, in the identity ( $\alpha$ ) the symbols $x$ by $c$ and making use of $(\beta)$,

$$
c_{\xi}^{2}\left[J_{6}-\frac{6 !}{5 !} J_{5} \lambda+\frac{6 !}{4 !} J_{4} \lambda^{2}-\frac{6 !}{3 !} J_{3} \lambda^{3}+\frac{6 !}{2 !} J_{2} \lambda^{4}-\frac{6 !}{1} J_{1} \lambda^{6}+6 ! \lambda^{6}\right] \equiv 0 \text {. }
$$

Hence $\lambda$ satisfies the equation

$$
\lambda^{6}-J_{1} \lambda^{5}+\frac{1}{2 !} J_{2} \lambda^{4}-\frac{1}{3 !} J_{3} \lambda^{3}+\frac{1}{4 !} J_{4} \lambda^{2}-\frac{1}{5 !} J_{5} \lambda+\frac{1}{6 !} J_{6}=0 .
$$

Returning to the original notation by introducing the factor $a_{\alpha}^{2}$ we have $I_{\kappa}$ expressed by means of the $\kappa$-rowed determinant

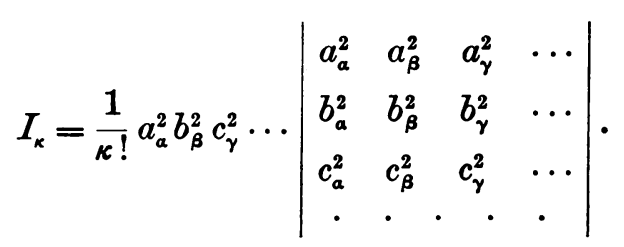

These properties of the invariants admit of immediate generalization and we have

Theorem $(A)$. Given two q-ary n-ics in complementary variables which, symbolically, are $f^{n} \equiv \alpha_{x: 1}^{n}=\alpha_{x: 2}^{n}=\cdots$ and $F^{n} \equiv a_{\xi: 1}^{n}=a_{\xi: 2}^{n}=\cdots$, there exist $N(\rho) q$-ary $\rho$-ics $(\rho \equiv n / \mathscr{2}), F^{p}$, such that the polar of $F^{p}$ as to $f^{n}$ has a polar as to $F^{n}$ which is identically $\lambda_{\iota} F^{\rho}$. These $N(\rho)$ quantities $\lambda_{\iota}$ satisfy 
an equation in $\lambda$ of degree $N(\rho)$ whose coefficients are simultaneous rational invariants of the same degree in the coefficients of $f^{n}$ and $F^{n}$.

If the coefficient of $\lambda$ to the highest power, be 1 , of the next power $-I_{1}$, etc., the general form of $I_{\kappa}$ is

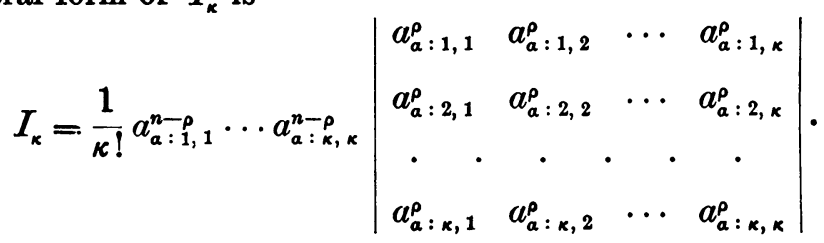

The series of invariants so formed closes with $I_{N(\rho)}, N(\rho)$ being the number of terms in the general $q$-ary $\rho$-ic.

Returning to the equation $\Delta_{2}^{4}(\lambda)=0$ for the case of two quartics, the vanishing of the constant term, i. e., the invariant $I_{6}=0$ is the condition that a polar conic (in this case, any conic) of the one shall be an apolar conic of the other and it breaks up into the product of the invariants $B$ formed for each quartic.

(VI) The various invariants in the equation $\Delta_{2}^{4}(\lambda)=0$ are the results of the successive performance of the operation

$$
\begin{aligned}
O \equiv \frac{2}{6}\left[\frac{\partial^{2}}{\partial a_{1111} \partial \alpha_{1111}}+\frac{1}{4} \frac{\partial^{2}}{\partial a_{1112} \partial \alpha_{112}}+\frac{1}{4} \frac{\partial^{2}}{\partial a_{1113} \partial \alpha_{1113}}+\frac{1}{6} \frac{\partial^{2}}{\partial a_{1122} \partial a_{1122}}\right. \\
\left.+\frac{1}{12} \frac{\partial^{2}}{\partial a_{1123} \partial \alpha_{1123}}+\cdots\right]
\end{aligned}
$$

upon the final invariant $I_{6}$.

For $O$ affects only the diagonal terms of $\Delta_{2}^{4}(0)$ and acting on each gives a result unity.

The only objection to the immediate extension of (VI) to the $\Delta_{\rho}^{n}(\lambda)$ equation is that the effect of the corresponding operator $O$ upon the various elements of the diagonal of $\Delta_{\rho}^{n}(0)$ is not obviously the same numerical constant for each element. This objection does not hold, for the general diagonal term is for ternary forms

$$
\frac{\rho !}{\kappa ! \lambda ! \mu !} a_{1}^{\kappa} a_{2}^{\lambda} a_{3}^{\mu} \alpha_{1}^{\kappa} \alpha_{2}^{\lambda} \alpha_{3}^{\mu} a_{a}^{n-\rho} \quad(\kappa+\lambda+\mu=\rho)
$$

Expanded, this becomes

$$
\sum \frac{\rho !}{\kappa ! \lambda ! \mu !} \frac{(n-\rho) !}{k ! l ! m !} a_{1}^{\kappa+k} a_{2}^{\lambda+l} a_{3}^{\mu+m} \alpha_{1}^{\kappa+k} \alpha_{2}^{\lambda+l} \alpha_{3}^{\mu+m},
$$

the summation extending to all values of $k, l, m$ such that $k+l+m=n-\rho$. The operator $O$ has its various terms affected by the reciprocals of the polynomial coefficients of the form of order $n$. The effect of operating upon the general diagonal term $(a)$ with $O$ is then 


$$
\sum_{k+l+m=n-\rho} \frac{\rho !}{\kappa ! \lambda ! \mu !} \frac{(n-\rho) !}{k ! l ! m !} \frac{(\kappa+k) !(\lambda+l) !(\mu+m) !}{n !} .
$$

The sum $(b)$ is independent of the choice of $\kappa, \lambda, \mu$ if $\kappa+\lambda+\mu=\rho$, i. e., (b) is independent of the particular diagonal term chosen. For the coefficient of $x^{k}$ in $(1-x)^{-\kappa-1}$ is

$$
\frac{(\kappa+k) !}{\kappa ! k !}
$$

Hence the coefficient of $x^{k} y^{l} z^{m}$ in the expansion of

$$
(1-x)^{-\kappa-1}(1-y)^{-\lambda-1}(1-z)^{-\mu-1} \quad \text { is } \quad \frac{(\kappa+k) !(\lambda+l) !(\mu+m) !}{k ! \kappa ! \lambda ! l ! \mu ! m !},
$$

then $(b)$ is to a factor $\rho !(n-\rho) ! / n !$ the sum of the coefficients of terms homogeneous and of degree $k+l+m=n-\rho$ in that expansion. For $z=y=x$, to the same factor, $(b)$ is the coefficient of $x^{n-p}$ in the expansion of $(1-x)^{-(\kappa+\lambda+\mu)-3}$, a coefficient independent of the choice of $\kappa, \lambda, \mu$, if always $\kappa+\lambda+\mu=\rho$. This proof is independent of the number of letters $k, l, m$ so that (VI) admits of extension also to forms with more variables. We have then

Theorem $(B)$. The invariant $I_{N(\rho)}$ of theorem $(A)$ when equated to zero is the condition that a polar $(n-\rho)$-ic of $f^{n}$ exist which $:: F^{n}$ and vice versa. If $\rho=n / 2, I_{N(\rho)}$ breaks up into two factors, each of which equated to zero gives the condition that one of the forms $f^{n}$ and $F^{n}$ have an apolar $\rho$-ic. The other invariants $I_{\kappa}$ of the series are the results of the successive application of the operator

$$
O \equiv c \sum_{k+l+m+\ldots=n} \frac{k ! l ! m ! \cdots}{(k+l+m+\cdots) !} \frac{\partial^{2}}{\partial a_{1}^{k} a_{2}^{l} \alpha_{3}^{m} \cdots \partial \alpha_{1}^{k} \alpha_{2}^{l} \alpha_{3}^{m} \ldots},
$$

where $c$ is a constant so adjusted as to make the result of $O$ upon a diagonal term of $\Delta_{\rho}^{n}(\lambda)$ unity.

Returning to the fixed conics of $f^{4}$ and $F^{4}$, their coefficients may be determined by putting $\lambda=\lambda_{\iota}$ and solving any five of the resulting equations. But for symmetry we may, following Hilbert, consider the modified system*

$$
\begin{aligned}
& c_{11}^{\iota}\left(a_{11} \alpha_{11} a_{a}^{2}-\lambda_{\iota}\right)+c_{22}^{\iota} a_{11} \alpha_{22} a_{a}^{2}+\cdots+c_{12}^{\iota} \cdot 2 a_{11} \alpha_{12} a_{a}^{2} \quad+x_{1}^{2} u=0, \\
& c_{11}^{\iota} a_{22} a_{11} a_{a}^{2}+c_{22}^{\iota}\left(a_{22} a_{22} a_{a}^{2}-\lambda_{\iota}\right)+\cdots+c_{12}^{\iota} \cdot 2 a_{22} a_{12} a_{a}^{2}+x_{2}^{2} u=0 \text {, } \\
& c_{11}^{\iota} a_{12} \alpha_{11} a_{a}^{2} \quad+c_{22}^{\iota} a_{12} \alpha_{22} a_{a}^{2} \quad+\cdots+c_{12}^{\iota}\left(2 a_{12} \alpha_{12} a_{a}^{2}-\lambda_{\iota}\right)+x_{1} x_{2} u=0, \\
& c_{11}^{\iota} \xi_{1}^{2}+c_{22}^{\iota} \xi_{2}^{2} \quad+\cdots+c_{12}^{\iota} \cdot 2 \xi_{1} \xi_{2} \quad-c_{\xi: \iota}^{2}=0 .
\end{aligned}
$$

* Here and below it seems desirable to use two notations interchangeably, writing the particular index of the $c_{i k}$ sometimes above, as in $c_{12}^{i}$, sometimes below as in $c_{\xi}^{2}:$. 
Solving for the unknowns $c_{i k}^{\iota}, u$ and $c_{\xi: \text {, we have }}^{2}$

$$
c_{11}^{\iota}: c_{22}^{\iota}: \cdots: c_{12}^{\iota}: u: c_{\xi: \iota}^{2}=\Delta_{c_{11}^{l}}: \Delta_{c_{22}^{l}}: \cdots: \Delta_{c_{12}^{l}}: \Delta_{u}: \Delta_{c_{\xi}^{2}: \iota},
$$

$\Delta_{\kappa}$ denoting the minors of the matrix of the system. Then $u=\Delta_{u}=\Delta_{2}^{4}\left(\lambda_{1}\right)=0$, i. e., the system (3) is equivalent to the original system. Putting

we have

$$
\Delta_{\mathrm{c}_{\xi}^{2}: \mathrm{l}}^{2} \equiv \Delta_{2}^{4}\left(x, \xi, \lambda_{\mathrm{\imath}}\right)
$$

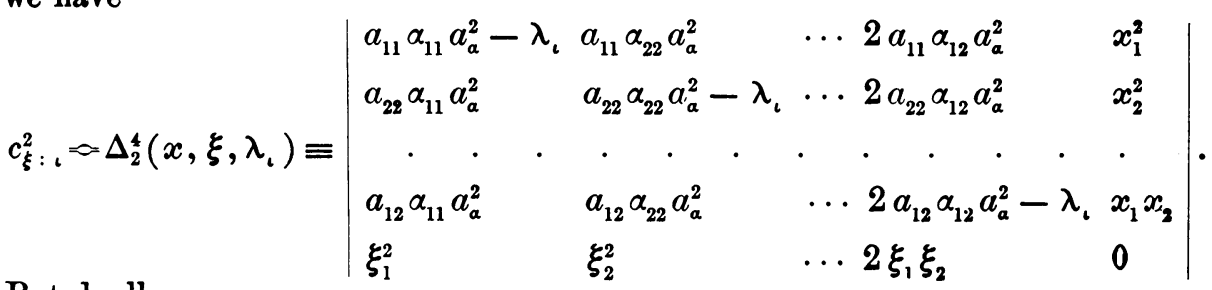

But dually

therefore

$$
\gamma_{x: \iota}^{2} \approx \Delta_{2}^{4}\left(x, \xi, \lambda_{\iota}\right) \text {, }
$$

$$
\Delta_{2}^{4}\left(x, \xi, \lambda_{\iota}\right) \approx \gamma_{\imath} c_{\imath} \text {. }
$$

Since $c_{\imath}$ on $f$ is $\gamma_{\iota}$ and $\gamma_{\imath}$ on $F$ is $c_{\iota}$ we have

$$
\left(\gamma_{\iota}\right)^{2} \approx \Delta_{2}^{4}\left(x, \alpha_{\iota \kappa} \alpha_{x}^{2}, \lambda_{\iota}\right) \text { and }\left(c_{\iota}\right)^{2} \approx \Delta_{2}^{4}\left(a_{\iota \kappa} a_{\xi}^{2}, \xi, \lambda_{\iota}\right) \text {. }
$$

These squares will appear in another connection. The bordered matrix $\Delta_{2}^{4}(x, \xi, \lambda)$ is a connex $(2,2)$ which for $\lambda=\lambda_{\iota}$ degenerates, i. e., breaks up into the product $c_{\xi: \iota}^{2} \cdot \gamma_{x: \iota}^{2} \cdot$ Arranged in powers of $\lambda$,

$$
\Delta_{2}^{4}(x, \xi, \lambda) \equiv K_{0} \lambda^{5}+K_{1} \lambda^{4}+\cdots+K_{5},
$$

where $K_{\mathrm{t}}$ is an invariantive connex $(2,2)$ of degree $\iota$ in the coefficients of $f^{4}$ and of $F^{4} . \quad K_{0}$ is $x_{\xi}^{2} . \quad K_{5}$ is $\Delta_{2}^{4}(x, \xi, 0)$ and for a given $x$ is the line conic whose polar as to $f$ has for polar as to $F$ the square of the point $x$, with a dual meaning for a given $\xi$.

And in general if the $\Delta_{\rho}^{n}(\lambda)$ of theorem $(A)$ be bordered with variables we obtain the connex $(\rho, \rho), \Delta_{\rho}^{n}(x, \xi, \lambda)$ which has properties entirely analogous to those of $\Delta_{2}^{4}(x, \xi, \lambda)$.

Neither is the generality of theorem $(A)$ diminished by the assumption $\rho \equiv n / 2$, for the fixed $(n-\rho)$-ics are the polars of the fixed $\rho$-ics as to $f^{n}$ and $F^{n}$.

We have found in the preceding section that under certain conditions $f^{4}$ and its contravariant $\phi$ possessed an infinite number, a pencil or even a web of fixed conics. For every fixed conic of $f^{4}$ and $F^{4}$ the identity (1) holds in which $\lambda$ is a root of $\Delta_{2}^{4}(\lambda)=0$. But $\Delta_{2}^{4}(\lambda)=0$ has always six roots, some of which may coincide. Having substituted a root in (1) the fixed copic is in gen- 
eral uniquely determined by the resulting equations. If, however, the chosen root $\lambda_{1}$ is such as to make every five-rowed minor of $\Delta_{2}^{4}(\lambda)$ vanish, the fixed conic may be determined from any four equations but no longer uniquely. Instead of using four equations consider the system (3) further modified by the addition to each of the first six equations of a term $y_{\imath} y_{\kappa} v$ and the adjunction of the equation $c_{\eta: \iota}^{2}=0$.

Then $u=\Delta_{2}^{4}\left(y, \eta, \lambda_{\iota}\right)$ and $v \approx \Delta_{2}^{4}\left(x, \eta, \lambda_{\iota}\right)$, both of which are identically zero, i. e., the modified system is equivalent to the original system. Further, $c_{\xi: \text { : }}^{2} \approx \Delta_{2}^{4}\left(x, y, \xi, \eta, \lambda_{\iota}\right)$ where

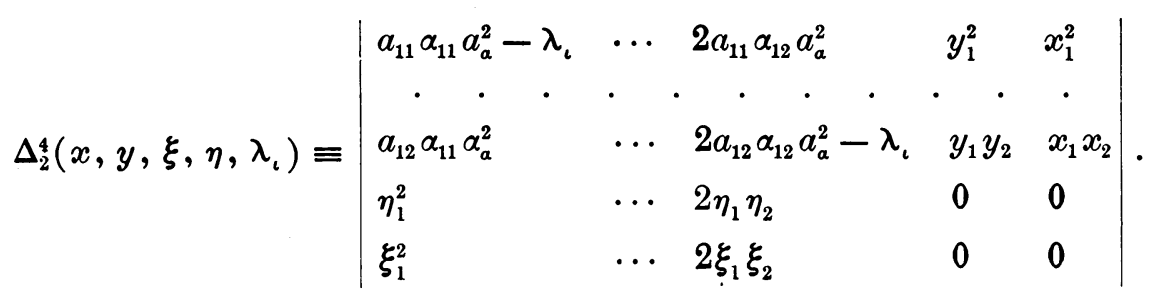

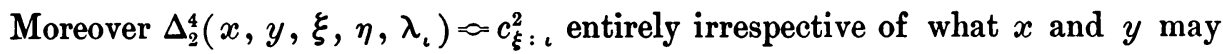
be and therefore $=c_{\xi: \imath}^{2} \cdot \phi(y, x)$. From the dual nature of the problem must then

$$
\Delta\left(x, y, \xi, \eta, \lambda_{\iota}\right) \approx c_{\xi: \iota}^{2} \cdot \gamma_{x: \iota}^{2} .
$$

The adjunction of the equation $c_{\eta: \iota}^{2}=0$ requires that $c_{\xi: \iota}^{2}$, touch the line $\eta$. Hence corresponding to a root $\lambda_{1}$ of $\Delta_{2}^{4}(\lambda)=0$ which makes all the five-rowed minors of $\Delta_{2}^{4}$ vanish, there exists a whole pencil of conics $c_{\iota}$; for to any given

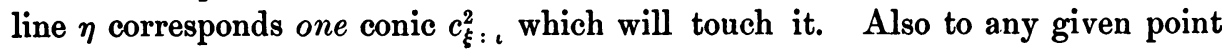
$y$ corresponds one conic $\gamma_{\iota}$ which passes through it. Such a root $\lambda_{\iota}$ is necessarily a double root of $\Delta_{2}^{4}(\lambda)=0$, though to a double root only one fixed conic may correspond.

In the same way if a root of $\Delta_{2}^{4}(\lambda)$, necessarily a triple root, makes all fourrowed minors vanish there will correspond to this root a web of line conics and a net of point conies, the product of the web and net being given by the thrice bordered determinant, $\Delta_{2}^{4}\left(x, y, z, \xi, \eta, \zeta, \lambda_{\iota}\right)$.

If finally a sextuple root of $\Delta_{2}^{4}(\lambda)$ makes all the elements of $\Delta_{2}^{4}(\lambda)$ vanish, the root is necessarily the bilinear invariant of $f$ and $F$. Every conic will be a fixed conic and $f$ and $F$ will stand in the reciprocal relation of $f$ and $\phi$ discussed in $\S 1$. All these bordered determinants possess the same invariantive character as $\Delta_{2}^{4}(x, \xi, \lambda)$.

The degenerations of $\Delta_{1}^{4}(\lambda)$, (and in general of $\Delta_{\rho}^{n}(\lambda)$ ) are entirely similar to those of $\Delta_{2}^{4}(\lambda)$. However for the general quartic $f^{4}$ infinitely many $F^{4}$ exist for which every point and line are "fixed." Among these are every $C_{504}$ of $f$. For let 
$f=\alpha_{x}^{4}=\beta_{x}^{4} ; \quad \sigma \equiv(\alpha \beta \xi)^{4} \equiv a_{\xi}^{4}=b_{\xi}^{4} ; \quad \bar{S} \equiv(a b \xi)^{4} ; \quad \bar{\phi} \equiv(\alpha \bar{S} \xi)^{4}, \quad A \equiv a_{a}^{4}$, then according to Marsano (Giornale di Mat., vol. 19) every $C_{504}$ belongs to the pencil $A \sigma-\lambda \bar{\phi}$. The final polar of a point $p_{\xi}$ as to $f$ and the pencil is

$$
A p_{a} a_{a}^{3} a_{\xi}+\lambda p_{a}(\alpha \beta \bar{S})^{3}(\beta \overline{S \xi}) \equiv A p_{a}(\alpha \beta \gamma)^{3}(\beta \gamma \xi)+\lambda p_{a}(a b c)^{3}(\beta \overline{S \xi}) .
$$

MaIsano shows that every symbolic expression which contains the apparent factors $(\alpha \beta \gamma)^{3}$ or $(a b c)^{3}$ contains the actual factors $(\alpha \beta \gamma)^{4}$ or $(a b c)^{4}$ respectively. Hence the final polar is of the form

$$
p_{\xi}\left[\mu A(\alpha \beta \gamma)^{4}+\nu(a b c)^{4}\right] .
$$

We have proved then (III) of $\S 1$. Also

(VII) $B=0$ is the condition that every polar cubic of $f:: \phi$ and vice versa. The corresponding fact for $f, \sigma$ and $A=0$ is stated by MaIsano (loc. cit.).

§3. Systems of Conics Connected with the Quartic.

To a certain extent point and line conics are related to a given quartic $f$ as are points and lines to a given conic. Thus

(I) If $c_{\xi: 1}^{2}::$ polar conic of $c_{\xi: 2}^{2}, c_{\xi: 2}^{2}::$ polar conic of $c_{\xi: 1}^{2}$. For, by the hypothesis, $c_{\xi: 1}^{2} \cdot c_{\xi: 2}^{2}:: f$, a perfectly mutual relation between $c^{1}$ and $c^{2}$.

The system of conics

$$
\left.\begin{array}{l}
\gamma_{x: 1}^{2}, \gamma_{x: 2}^{2}, \cdots, \gamma_{x: 6}^{2} \\
c_{\xi: 1}^{2}, c_{\xi: 2}^{2}, \cdots, c_{\xi: 6}^{2}
\end{array}\right\}
$$

shall be called an apolar system if $\gamma_{x: \iota}^{2}:: c_{\xi: \kappa}^{2}$ for $\iota \neq \kappa$ and $\gamma_{x: \iota}^{2}$ not $:: c_{\xi: \iota}^{2}$. Such a system is an immediate extension of the three points and three lines which form a triangle and may be obtained by arbitrarily choosing six linearly independent point conics. The line conics will then be determined by the apolarity conditions. Thus taking point conics with, line conics without, polynomial coefficients we have

$$
c_{\xi: \kappa}^{2}=\Gamma_{11}^{\kappa} \xi_{1}^{2}+\Gamma_{23}^{\kappa} \xi_{2} \xi_{3}+\cdots,
$$

where $\Gamma_{m n}^{k}$ is the minor of $\gamma_{m n}^{k}$ in

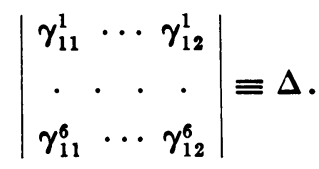

II. If in two systems each consisting of six point conics and six line conics the point conics of each are the polars, as to a quartic, of the line conics in the other system, then if either one is an apolar system, so also is the other. 
This follows immediately from (I).

In a certain sense the one apolar system is the polar of the other. If it coincides with its polar the system will be called a "self-polar system" of $f$. In a self polar system certain relations exist among the conics, determined as follows:

If the polar of $C_{\xi: \kappa}^{2}$, i. e., $\Gamma_{\xi: \kappa}^{2}$ as to $f$ is $\lambda_{\kappa} \gamma_{x: \kappa}^{2}$ then

$$
\Gamma_{a: \kappa}^{2} \alpha_{x}^{2} \equiv \lambda_{\kappa} \gamma_{x: \kappa}^{2} \quad(\kappa=1,2, \cdots, 6) .
$$

Equating coefficients of $x_{\imath} x_{j}$ for $\kappa=1, \ldots, 6$ we have a system of six non-homogeneous equations whose determinant is the adjoint of $\Delta$ and whose solution gives, to a factor $1 / \Delta$,

$$
\alpha_{i j, m n}=\sum_{1=\rho}^{6} \lambda_{\rho} \gamma_{i j}^{\rho} \gamma_{m n}^{\rho}
$$

Hence already $\alpha_{\iota \kappa, l m}=\alpha_{l m, \iota \kappa}$. But necessarily

Therefore

$$
\alpha_{1122}=\alpha_{1212} \text { and } \alpha_{1123}=\alpha_{1213}, \cdots
$$

etc.

$$
\begin{gathered}
\lambda_{1}\left(\gamma_{11}^{1} \gamma_{22}^{1}-\gamma_{12}^{2} \gamma_{12}^{2}\right)+\cdots+\lambda_{6}\left(\gamma_{11}^{6} \gamma_{22}^{6}-\gamma_{12}^{6} \gamma_{12}^{6}\right)=0, \\
\lambda_{1}\left(\gamma_{11}^{1} \gamma_{23}^{1}-\gamma_{12}^{1} \gamma_{13}^{1}\right)+\cdots+\lambda_{6}\left(\gamma_{11}^{6} \gamma_{23}^{6}-\gamma_{12}^{6} \gamma_{13}^{6}\right)=0,
\end{gathered}
$$

If the point conics have been chosen as repeated lines, equations (3) are identically satisfied. If not, they mean that a linear identity

$$
\sum_{1=\rho}^{6} \lambda_{\rho}\left(\gamma_{\rho} \gamma_{\rho}^{\prime} \xi\right)^{2} \equiv 0
$$

exists among the line equations of the point conics.

(III) If an apolar system is a self-polar system of a quartic $f$, a linear identity must necessarily exist among the line equations of the point conics.

These self-polar systems may be constructed for the given quartic $f$ as follows: Take any conic $c_{\xi: 1}^{2}$ and its polar, $\gamma_{x: 1}^{2}$; take $c_{\xi: 2}^{2}:: \gamma_{x: 1}^{2}$, otherwise arbitrary, and its polar $\gamma_{x: 2}^{2} ;$ take $c_{\xi: 3}^{2}:: \gamma_{x: 1}^{2}$ and $\gamma_{x: 2}^{2}$ and linearly independent of $c_{1}$ and $c_{2}$, and its polar $\gamma_{x: 3}^{2}$. Continuing in this way up to 5 we have finally $\dot{c}^{6}$ completely determined by the five preceding $\gamma$ 's. Its polar $\gamma^{6}$ is also apolar to the five preceding $c$ 's by (I). In the choice of the conics $c$ such must be avoided as are apolar to their own polar, else linear identities will exist among the conics. The choice of the system depends then on 15 constants. By (III), a linear identity will exist among the line equations of the point conics. Relations will also exist among the coefficients of the line conics, but these are of higher order. 
(IV) The quartic $f$ is linearly expressible as a sum of the squares of the point conics of any self-polar system.

For a self-polar system of $f$, the necessary relations $(\S 3,(3))$ hold. From (2) then, theorem (IV) follows at once.

This includes the expression of the quartic as a sum of the fourth powers of linear forms. For it is only necessary to chose the point conics as the squares of lines. The manner of choice is also indicated by the theorem. The first line is chosen at random. Since the square of the second line is to be apolar to the associate conic of the first it must touch it and for the same reason the four other lines must be the four common tangents of the first two associate conics. Also the first two lines must not be self-associate, as was previously noted for the general self-polar system (see ScherRer, loc. cit.).

(V) A point quartic $f$ is expressible as a sum of squares of six point conics, and simultaneously a line quartic $F$ as a sum of squares of six line conics, the conics forming a self-polar system of both $f$ and $F$. This reduction is unique.

For, from $\S 2$, the two quartics possess one and only one common self-polar system.

The following facts may now be noted:

(a) Having fixed the homogeneous coefficients in a self-polar system of $f$, in the expression

$$
f=\sum_{1}^{6} \rho_{\kappa}\left(\gamma_{x: \kappa}^{2}\right)^{2}
$$

the coefficients $\rho_{\kappa}$ are such that, to a factor independent of $\kappa,\left(c_{\xi: \kappa}^{2}\right.$ on $\left.f\right) \equiv \rho_{\kappa} \gamma_{x: \kappa}^{2}$.

(b) The coefficients in the linear identity of $\S 3$, (III) are these same $\rho$ 's.

(c) The polar reciprocal of $c_{\xi}^{2}$ as to $\gamma_{x}^{2}\left(\equiv \gamma_{x}^{\prime 2}\right)$ is

$$
\gamma_{c} \gamma_{c}^{\prime} \gamma_{x} \gamma_{x}^{\prime} \equiv P_{c, \gamma} \text {. }
$$

(d) The intermediate of $c_{\xi}^{2}$ and $\left(\gamma \gamma^{\prime} \xi\right)^{2}$ is

$$
c_{\gamma}^{2} \gamma_{x}^{2}-P_{c, \gamma} \equiv I_{c, \gamma}
$$

(e) The polar of $c_{\xi}^{2}$ as to $\left(\gamma_{x}^{2}\right)^{2}$ is

$$
\begin{gathered}
\gamma_{c}^{2} \gamma_{x}^{2}+2 P_{c, \gamma}=3 \gamma_{c}^{2} \gamma_{x}^{2}-2 I_{c, \gamma} . \\
\rho_{1} I_{c, \gamma_{1}}+\rho_{2} I_{c, \gamma_{2}}+\cdots=I_{c, \rho_{1} \gamma_{1}+\rho_{2} \gamma_{2}+\ldots} .
\end{gathered}
$$

(g) $\gamma_{c: \kappa, \kappa}^{2}$ is the $\Delta$ of page 79 and is independent of $\kappa$.

Fixing the homogeneous coefficients of the conics of the common self-polar system of $f$ and $F$ we have 


$$
\begin{aligned}
& f \equiv \pi_{1}\left(\gamma_{x: 1}^{2}\right)^{2}+\cdots+\pi_{6}\left(\gamma_{x: 6}^{2}\right)^{2}, \\
& F \equiv \rho_{1}\left(c_{\xi: 1}^{2}\right)^{2}+\cdots+\rho_{6}\left(c_{\xi: 6}^{2}\right)^{2},
\end{aligned}
$$

where $\pi_{\kappa}$ and $\rho_{\kappa}$ are defined by $(a)$ and $(b)$.

By $(d),(e)$ and $(f)$ the polar of $c_{\xi: 1}^{2}$ as to $f$ is

$$
3 \pi_{1} \gamma_{c: 1,1}^{2} \gamma_{x: 1}^{2}-2 I_{c: \pi_{1} \gamma_{1}+\ldots+\pi_{6} \gamma_{\theta}, 1} \text {. }
$$

But by $(d), I_{c, \gamma}$ is formed with regard to the line equation of $\gamma$, and by $(b)$, $\sum \pi_{\kappa}\left(\gamma \gamma^{\prime} \xi\right)^{2} \equiv 0$; also by $(g), 3 \gamma_{c 1: 1}^{2}$ may be neglected and we have that the polar of $c_{\xi: 1}^{2}$ as to $f$ is $\pi_{1} \gamma_{x: 1}^{2}$. Similarly the polar of $\pi_{1} \gamma_{x: 1}^{2}$ as to $F$ is $\pi_{1} \rho_{1} c_{\xi: 1}^{2}$. Therefore

(VI) If according to (V) $\S 3$, the quartics $f$ and $F$ are put in the form $(5)$, the roots of the $\Delta_{2}^{ \pm}(\lambda)$ equation of $f$ and $F$ are such that, to a factor independent of $\kappa$,

We may also state

$$
\lambda_{\kappa}=\pi_{\kappa} \rho_{\kappa} \text {. }
$$

(VII) Any two point quartics $f$ and $f^{\prime}$ are simultaneously expressible in but one way as a sum of squares of the same six point conics, the associate conics of these point conics being the same for both $f$ and $f^{\prime}$, and the point and associate conics together forming a self-polar system of both $f$ and $f^{\prime}$. Two distinct linear identities exist among the line equations of the point conics.

Theorem (III) gives a necessary condition that an apolar system be a selfpolar system of a quartic. No other conditions subsist. For a general apolar system depends on 30 constants. If it be a self-polar system of some (undetermined) quartic, the line equation of one point conic is determined linearly in terms of the other five; this cuts out five constants. However, the ratios of five coefficients in the identity which gives the one in terms of the other introduce four more constants. A self-polar system of an undetermined quartic involves therefore 29 constants. But for a given quartic a self-polar system depends on 15 constants; these added to the 14 arbitrary constants involved in the given quartic make up the requisite 29 .

Included in the aggregate of self-polar systems of $f(15$ times infinite) is an aggregate (14 times infinite) of systems which $f$ has in common with line quartics $F$ and an aggregate (also 14 times infinite) which $f$ has in common with other point quartics $f^{1}$.

\section{§4. Conics in a Five-dimensional Space.}

It is of interest to derive some of the foregoing results from the representation of conics in the plane $S_{2}$ by means of a five-dimensional space $S_{5}$. This repre- 
sentation has been developed by STUDY and others.* Use will be made chiefly of STUDY's work.

To line conics $c, c_{1}, c_{2}, \ldots$ in $S_{2}$ correspond in $S_{5}$ respectively points $p, p_{1}, p_{2}, \cdots$; to point conics $\gamma, \gamma_{1}, \gamma_{2}, \ldots$ in $S_{2}$ correspond in $S_{5}$ respectively flat spaces $\sigma, \sigma_{1}, \sigma_{2}, \ldots$; to apolar $\gamma$ and $c$ in $S_{2}$ correspond in $S_{5}$ incident $\sigma$ and $p$. Denote the general $p$-manifold in $S_{b}$ of dimensions $k$ and order $l$ is $M_{\kappa}^{l}$; the $\sigma$-envelope $M_{\kappa}^{l}$. To point pairs in $S_{2}$ correspond in $S_{5}$ the $p$ 's of an $M_{4}^{3} \equiv L^{3} ;$ on this lies an $M_{2}^{4} \equiv F_{2}^{4}$, the $p$-locus which corresponds to repeated points in $S_{2}$. Dually, to line pairs in $S_{2}$ correspond in $S_{5}$ the $\sigma$ 's of an $\mathbf{M}_{4}^{3} \equiv \Lambda^{3}$ which is inscribed to an $M_{2}^{4} \equiv \phi_{2}^{4}$, the $\sigma$-envelope which corresponds to repeated lines in $S_{2}$. The surface $F_{2}^{4}$ occurs as double points in $L^{3}$, and $\Lambda^{3}$ contains the envelope $\phi_{2}^{4}$ as double $\sigma$ 's. Hence the polar quadric of any $p$ as to $L^{3}$ contains $F_{2}^{4}$; the polar quadric of any $\sigma$ as to $\Lambda^{3}$ is inscribed to $\phi_{2}^{4}$.

(I) Every quadric $M_{4}^{2}$ containing $F_{2}^{4}$ is a polar quadric of $L^{3}$ and may be put in the form $\lambda_{1} F_{1}+\cdots+\lambda_{6} F_{6}$ where $F_{1}, \cdots, F_{6}$ are linearly independent polar quadrics. Every quadric $\mathbf{M}_{2}^{4}$ inscribed to $\phi_{2}^{4}$ is a polar quadric of $\Lambda^{3}$ and may be put in the form $\lambda_{1} \phi_{1}+\cdots+\lambda_{6} \phi_{6}$ where $\phi_{1}, \cdots, \phi_{6}$ are linearly independent polar quadrics.

If the second polar of $p_{1}$ as to $L^{3}$ is $\sigma_{1}$, then the $\gamma_{1}$ of $p_{1}$ is the reciprocal of the $c_{1}$ of $\sigma_{1}$. If the second polar of $\sigma_{1}$ as to $\Lambda^{3}$ is $p_{1}$, then $c_{1}$ is the reciprocal of $\gamma_{1}$. This coördination of $p$ and $\sigma$ in $S_{5}$ which corresponds in $S_{2}$ to the same conic in complementary variables is equivalent to a dual quadratic Cremona transformation, $\mathrm{T}$, with singular surfaces $F_{2}^{4}$ and $\phi_{2}^{4}$.

Also if the mixed polar of $p_{1}$ and $p_{2}$ as to $L^{3}$ is $\sigma_{1}$, then $\gamma_{1}$ is the intermediate of $c_{1}$ and $c_{2}$. If the polar of $\sigma_{1}$ and $\sigma_{2}$ as to $\Lambda^{3}$ be $p_{1}$ then $c_{1}$ is the intermediate of $\gamma_{1}$ and $\gamma_{2}$.

Study (Mathematische Annalen, vol. 27, p. 87) proves that

(II) If an $M_{4}^{l}$ contains $F_{2}^{4} \lambda$ times and an $M_{4}^{l^{\prime \prime}}$ contains $\phi_{2}^{4} \lambda^{\prime}$ times and further $M_{4}^{l}$ and $\mathbf{M}_{4}^{l \prime}$ correspond to each other by the transformation $T$, then between the numbers $l, l^{\prime}, \lambda, \lambda^{\prime}$ these equations exist :

$$
\begin{array}{ll}
l^{\prime}=2 l-3 \lambda, & l=2 l^{\prime}-3 \lambda^{\prime}, \\
\lambda^{\prime}=l-2 \lambda, & \lambda=l^{\prime}-2 \lambda^{\prime} .
\end{array}
$$

If then $l^{\prime}=2$ and $\lambda^{\prime}=1, l=1$ or we have the

Corollary: An $\mathrm{M}_{4}^{2}$ inscribed to $\phi_{2}^{4}$ is transformed by $T$ into a flat space $\sigma$, i. e., every $\sigma_{\kappa}$ of the quadric $\mathbf{M}_{4}^{2}$ goes by the dual transformation $T$ into a point $p_{\kappa}$ which lies in $\sigma$.

Study (Mathematische Annalen, vol. 40, p. 574) proves further

* Study, Mathematische Annalen, vols. 27 (1886) and 40 (1892). Segre, Atti Aoo. Torino, vol. 20 (1885). 
(III) Through a curve of order $4 l$ lying on $F_{2}^{4}$ may be passed a SLNGLE $M_{4}^{l}$ such that every quadric $\mathbf{M}_{4}^{2}$ inscribed to $\phi_{2}^{4}$ is apolar to $M_{4}^{l} \cdot{ }^{*}$

To curves of order $l$ in $S_{2}$ (considered as made up of repeated points) correspond on $F_{2}^{4}$ curves of order $2 l$. Given then a point quartic $f$ in $S_{2}$. To it corresponds on $F_{2}^{4}$ a curve of order 8 which is cut out by a single quadric $Q$ of the type described in (III). This same curve however is cut out of $F_{2}^{4}$ by $\infty^{6}$ quadrics $Q_{1}^{\prime}, Q_{2}^{\prime}, \ldots$ But of all these, $Q$ is the only one whose polar system represents the polar system of $f$ in $S_{2}$. Then to the self-polar " bases" or " tetrahedroids" of $Q$ correspond the self-polar systems of $f$ treated in $\S 3$.

(IV) Given an $M_{4}^{2}$ and $\mathbf{M}_{4}^{2}$ in $S_{5}$, the necessary and sufficient condition that $M_{4}^{2}:: \mathbf{M}_{4}^{2}$ is that when five $\sigma$ 's of a basis of $\mathbf{M}_{4}^{2}$ touch $M_{4}^{2}$, the sixth also touches $M_{4}^{2}$.

The proof is entirely analogous to the proof of the corresponding theorem for two conics in $S_{2}$.

Having chosen a self-polar basis of the particular quadric $Q$ which cuts out of $F_{2}^{4}$ the quartic $f$, by (I) a polar quadric of $\Lambda^{3}$ may be found which touches five of the $\sigma$ 's of this basis. From (III) $Q$ :: this polar quadric which by (IV) will then touch all six $\sigma$ 's of the basis. Since this polar quadric is also inscribed to $\phi_{2}^{4}$, according to (II) Corollary, these six $\sigma$ 's of the basis are transformed by $\mathrm{T}$ into points of a certain $\sigma$, i. e., among the six points a linear identity exists. Hence

(V) Among the line equations of the point conics of a self-polar system of $f$ a linear identity exists. . [See $§ 3$ (III).]

As has been said, $\infty^{6}$ quadrics $Q_{1}^{\prime}, Q_{2}^{\prime}, \ldots$ cut $F_{2}^{4}$ in the same curve as $Q$. Among the polar systems of these quadrics certain relations exist. Denoting any two by $Q_{1}^{\prime}$ and $Q_{2}^{\prime}$, every member of the pencil $Q_{1}^{\prime}+\lambda Q_{2}^{\prime}$ also cuts $F_{2}^{4}$ in that curve and in general only in that curve. But by a proper choice of $\lambda$, say $\lambda_{0}$, one of the pencil may be made to go through an additional point of $F_{2}^{4}$, i. e., to contain $F_{2}^{4}$; therefore

$$
Q_{1}^{\prime}+\lambda_{0} Q_{2}^{\prime} \equiv \bar{Q}_{1},
$$

denoting by $\bar{Q}_{1}$ a quadric which contains $F_{2}^{4}$. By virtue of this identity we have an identity of polar systems or

$$
\left(p \text { on } Q_{1}^{\prime}\right)+\lambda_{0}\left(p \text { on } Q_{2}^{\prime}\right) \equiv\left(p \text { on } \bar{Q}_{1}\right) .
$$

But $\bar{Q}_{1}$ is the polar quadric of some point say $p_{1}$ as to $L_{4}^{3}$. Hence

$$
\left(p \text { on } Q_{1}^{\prime}\right)=-\lambda_{0}\left(p \text { on } Q_{2}^{\prime}\right)+\left(p p_{1} \text { on } L_{4}^{3}\right) \text {. }
$$

Since $p p_{1}$ on $L_{4}^{3}$ gives the intermediate of $p$ and $p_{1}$ we have

* The representation of conics just described together with Theorems I, II and III are taken directly from the cited works of STUDY. What follows is an application of STUDY's methods to the proof of some of the results obtained in $\S \S 1$ and 2 . 
(VI) The polar systems of $Q_{1}^{\prime}$ and $Q_{2}^{\prime}$, any two quadrics of the system $Q^{\prime}$ (which includes the special quadric $Q$ ) coincide to within an additive term, viz., the $\sigma$ which is the intermediate of a fixed point $p_{1}$ (fixed except as to variation with $Q_{1}^{\prime}$ and $Q_{2}^{\prime}$ ) and the variable point $p$ whose polar is taken.

The reciprocal of $Q$ is an $\mathbf{M}_{4}^{2}, q$, intersecting $\phi_{2}^{4}$ in $\sigma$ 's which correspond to the lines of the contravariant of $f$ denoted in $\S 1$ by $\phi$.

(VII) In general $q$ is not : : every point quadric containing $F_{2}^{4}$. If it is, $f$ must be the "special " quadric of $\S 1$.

But by (III) there is a quadric $\bar{q}$ which cuts $\sigma_{2}^{4}$ in the same $\sigma$ 's as $q$ and whose polar system represents that of the contravariant $\phi$. Applying to $q$ and $\bar{q}$ the dual of proposition (VI) we have the formula :

$$
(\gamma \text { on } \phi)=I_{\gamma}+\lambda A_{\gamma} \quad(\text { See } \S 1(\mathrm{XI})) .
$$

The covariant conic $C$ is then the dual of the fixed point $p_{1}$ in (VI).

The polar system of the quadric $q$ is represented in $S_{2}$ by the quadri-quadric form $\phi(\xi, \eta)$ (see footnote, $\S 1$ ).

This treatment of the quartic as a quadric in $S_{5}$ has the further advantage of indicating clearly all possible expressions of the quartic as a sum of squares of six conics. The expression by means of "self polar systems" is obtained only by means of the bases of the particular quadric $Q$. But every basis of any of the $\infty^{6}$ quadrics $Q_{1}^{\prime}, Q_{2}^{\prime}, \ldots$ also gives rise to an expression of the quartic as a sum of squares. An enumeration of the constants will verify this. As to the quartic and its contravariant $\phi$ we have then

(IX) The contravariant $\phi$ is expressible as a sum of squares of the line conics of EVERY self polar system of the quartic $f$.

This system of quadrics in $S_{5}$ may also be serviceable in the solution of further problems with regard to the quartic. For example, the quartic $f$ has its contravariant $\phi, \phi$, its covariant $f^{\prime}$, etc. It would be an advance in the theory of the quartic to determine whether this series of quartics $f, f^{\prime}$, etc., ever closes. 